\title{
The Cytokine Expression in Patients with Cardiac Complication after Immune Checkpoint Inhibitor Therapy
}

\author{
Toshihiro Tsuruda ${ }^{1}$, Naoki Yoshikawa ${ }^{2}$, Motoaki Kai ${ }^{1}$, Masashi Yamaguchi ${ }^{1}$, Reiko Toida ${ }^{3}$, \\ Tsuyoshi Kodama ${ }^{4}$, Kei Kajihara ${ }^{5}$, Takayuki Kawabata ${ }^{5}$, Takeshi Nakamura ${ }^{5}$, Koji Sakata ${ }^{1}$, \\ Kinta Hatakeyama ${ }^{6}$, Toshihiro $\mathrm{Gi}^{7}$, Yujiro Asada ${ }^{7}$, Tetsuya Tono ${ }^{5}$,
}

Kazuo Kitamura ${ }^{1}$ and Ryuji Ikeda ${ }^{2}$

\begin{abstract}
:
We herein report the cytokine expression at different stages for three patients who developed cardiac complications after immune checkpoint inhibitor (ICI) therapy. Case 1 with biopsy-proven myocarditis showed increased levels of interleukin (IL)-8, monocyte chemotactic and activating factor, and granulocyte macrophage colony-stimulating factor (GM-CSF) when he developed Takotsubo cardiomyopathy. Case 2 with subclinical myocarditis showed predominant activation of IL-8 during the progressive clinical course. Case 3 with cytokine-releasing syndrome showed substantial activations of IL-6, IL-8, GM-CSF, and interferon- $\gamma$. Our data suggest the development of unique cytokine activation in individual patients with cardiac complications after ICI therapy.
\end{abstract}

Key words: cancer, myocarditis, cytokine releasing syndrome, troponin

(Intern Med 60: 423-429, 2021)

(DOI: 10.2169/internalmedicine.5317-20)

\section{Introduction}

Cytokines are soluble proteins roughly 5 to $20 \mathrm{kDa}$ in size that regulate host immune activity through a highly complex network. With only a limited half-life, they work as pro-inflammatory or anti-inflammatory signals by binding high-affinity receptors $(1,2)$. The cytokine network can contribute to the pathogenesis of a number of disorders, such as bronchial asthma, inflammatory bowel disease, and rheumatoid arthritis (3-5). The discovery of immune checkpoints, such as cytotoxic T-lymphocyte-associated antigen 4 , and programmed cell death protein 1 (PD-1)/programmed cell death ligand 1 (PD-L1), has advanced our understanding of immunity. As a result, immune checkpoint inhibitors (ICIs) have brought about a new era in cancer therapy (6).
ICIs activate T-cell proliferation and exert anti-tumor immunity but also induce a wide spectrum of immune-related adverse effects (irAEs) via the release of cytokines [e.g., tumor necrosis factor (TNF)- $\alpha$, interferon (IFN)- $\gamma$, granulocyte macrophage colony-stimulating factor (GM-CSF), interleukin (IL)-1 $\alpha$, and IL-1 $\beta](7,8)$. irAE-related myocarditis is reported with frequency between 18/20,594 (0.09\%) and $11 / 964(1.14 \%)(9,10)$, with wide variation in severity (from smoldering to fulminant myocarditis) $(11,12)$.

Circulatory cytokine concentrations are increased in viral myocarditis and cardiomyopathy $(13,14)$, so we measured the serum cytokine concentrations of three cancer patients with cardiac complications after ICI therapy at different stages in their clinical course. The present study explored the activation of cytokines associated with cardiac complications after ICI therapy. In addition, we postulated that in-

\footnotetext{
${ }^{1}$ Department of Internal Medicine, Circulatory and Body Fluid Regulation, Faculty of Medicine, University of Miyazaki, Japan, ${ }^{2}$ Department of Pharmacy, University of Miyazaki Hospital, Japan, ${ }^{3}$ Department of Cardiology, Chiyoda Hospital, Japan, ${ }^{4}$ Department of Respiratory Medicine, Chiyoda Hospital, Japan, ${ }^{5}$ Department of Otolaryngology, Head \& Neck Surgery, Faculty of Medicine, University of Miyazaki, Japan, ${ }^{6}$ Department of Pathology, National Cerebral and Cardiovascular Center, Japan and ${ }^{7}$ Department of Pathology, Faculty of Medicine, University of Miyazaki, Japan

Received: May 11, 2020; Accepted: June 25, 2020; Advance Publication by J-STAGE: September 19, 2020

Correspondence to Dr. Toshihiro Tsuruda, ttsuruda@med.miyazaki-u.ac.jp
} 
creased levels of cytokines at certain time points may predict immune-related cardiac toxicity.

\section{Materials and Methods}

Residual serum samples were collected as a by-product of daily clinical biochemistry testing and stored at $-80^{\circ} \mathrm{C}$ until a cytokine analysis could be performed. We measured IL-1 $\alpha$, IL-1 $\beta$, IL-6, IL-8, GM-CSF, IFN- $\gamma$, monocyte chemotactic and activating factor (MCAF), and TNF- $\alpha$ simultaneously with a pro-inflammatory multiplex ELISA Kit (Cat No. CK500900; Antigenix America, Huntington Station, USA). The major functions of these cytokines are as follows (2): IL- $1 \alpha$ and IL-1 $\beta$, pyrogenic, pro-inflammatory, proliferation and differentiation, bone marrow cell proliferation; IL-6, differentiation into plasma cells, IgG production; IL-8, chemotaxis, pro-inflammatory; MCAF (also called monocyte chemotactic factor-1), chemotaxis, angiogenesis; IFN- $\gamma$, antiviral, macrophage activation, an increased neutrophil and monocyte function, major histocompatibility complex-I and complex-II expression on cells; TNF- $\alpha$, phagocyte cell activation, endotoxic shock; and GM-CSF, granulocyte, monocyte, and eosinophil production.

In addition, we measured the concentrations of pembrolizumab and nivolumab with an ELISA Kit (C-PE01Q, C-NI 01Q; Metallogenics, Chiba, Japan).

\section{Case Reports}

\section{Case 1}

A 75-year-old man developed subclinical myocarditis (grade 1 irAEs) on the 21 st day after his first pembrolizumab (200 mg) infusion for recurrent squamous cell carcinoma of the lung. He had had his left-upper lobe and regional lymph nodes resected 27 months earlier and undergone 4 administrations of cisplatin and vinorelbine. He was admitted to our hospital due to an elevated creatine kinase level. However, he did not show any clinical signs of acute coronary syndrome or heart failure. His temperature was $35.8^{\circ} \mathrm{C}$, blood pressure $140 / 92 \mathrm{mmHg}$, and heart rate 80 beats per minute. Chest X-ray revealed a cardiothoracic ratio of $45 \%$ without congestion in the lung fields. A 12-lead electrocardiogram (ECG) exhibited sinus rhythm and a previously unrecognized, completely blocked right branch bundle (Fig. 1A). At the time of admission, blood tests showed elevated levels of creatine kinase at 2,859 U/L (reference range, 30-200 U/L), creatine kinase-MB isoenzyme at 117 $\mathrm{U} / \mathrm{L}$ (reference range, 0-12 U/L), and cardiac troponin-T at 0.37 ng/mL (Roche Diagnostics, Mannheim, Germany; reference range, $<0.014 \mathrm{ng} / \mathrm{mL}$ ). The pembrolizumab concentration in the blood was $7.77 \mu \mathrm{g} / \mathrm{mL}$. Magnetic resonance imaging showed a left ventricular ejection fraction of $57 \%$ (Fig. 1B-a, -b) and minimal myocardial edema (Fig. 1B-c) but displayed late gadolinium enhancement in the subepicardial regions of the mid- and lateral wall (Fig. 1B-d). Two endomyocardial biopsies performed at a one-month interval both showed active myocardial inflammation in hematoxylin-eosin staining (Fig. 1C-a, -f). In adjacent sections at the first biopsy, CD3 T-lymphocytes expressed PD-1 (Fig. 1C-b, -c), whereas CD68 macrophages expressed PD-L 1 (Fig. 1C-d, -e). A transthoracic echocardiogram displayed a left ventricular ejection fraction of $56 \%$ without regional wall motion abnormalities (Fig. 1D).

Monotherapy for methylprednisolone was continuously administered intravenously, and oral prednisolone was given followed by the methylprednisolone treatment. At admission, all cytokine values were negative except for MCAF (88 pg/ $\mathrm{mL})$. By the 115th hospital day, the concentrations were as follows: IL-6 (2,214 pg/mL), IL-8 (3,211 pg/mL), MCAF $(2,333 \mathrm{pg} / \mathrm{mL})$, and GM-CSF $(1,460 \mathrm{pg} / \mathrm{mL})$ (Fig. 1E). At that time, the 12-lead ECG revealed widespread T-wave inversion concomitant with QT prolongation, and the transthoracic echocardiogram displayed apical ballooning form in the left ventricle, suggesting Takotsubo cardiomyopathy. We did not perform coronary angiography.

Cytokines IL- $1 \alpha$, IL-1 $1 \beta$, IFN- $\gamma$, and TNF- $\alpha$ were not detected during the clinical course. The immune-suppressive treatment decreased the creatine kinase level, but troponin I (133 pg/mL, Abbott Japan, Tokyo, Japan, reference range < $30 \mathrm{pg} / \mathrm{mL}$ ) and C-reactive protein (CRP) levels increased as Takotsubo cardiomyopathy (Fig. 1F) and pericardial effusion progressed. The patient died on the 120th day at the affiliated hospital, possibly due to ventricular wall rupture. No autopsy was performed.

\section{Case 2}

A 47-year-old man suffering from ethmoid sinus cancer developed subclinical myocarditis (grade 1 irAEs) on the 16th day after his third nivolumab $(3 \mathrm{mg} / \mathrm{kg}$ ) infusion. He had been treated three times with cisplatin and irinotecan and received radiation. His temperature was $36.9^{\circ} \mathrm{C}$, blood pressure 130/82 mmHg, heart rate 90 beats per minute, and respiratory rate 17 breaths per minute. He had no signs of heart failure or acute coronary syndrome, and the 12-lead ECG displayed a sinus rhythm without ST-T segment changes (Fig. 2A).

At the time of admission, blood tests showed white-blood

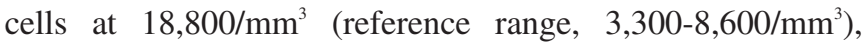
neutrophils at $90.3 \%$ (reference range, $37-72 \%$ ), platelet cell count at $70,000 / \mathrm{mm}^{3}$ (reference range 158,000-348,000/ $\mathrm{mm}^{3}$ ), creatine kinase at 3,385 U/L (reference range, 59-248 $\mathrm{U} / \mathrm{L}$ ), creatine kinase-MB isoenzyme at 1,221 U/L (reference range, $<12 \mathrm{U} / \mathrm{L}$ ), cardiac troponin- $\mathrm{T}$ at $3.30 \mathrm{ng} / \mathrm{mL}$ (reference range, $<0.014 \mathrm{ng} / \mathrm{mL}$ ), and CRP at $0.50 \mathrm{mg} / \mathrm{dL}$ (reference range, $0-0.14 \mathrm{mg} / \mathrm{dL}$ ). The nivolumab concentration was $3.45 \mu \mathrm{g} / \mathrm{mL}$ in the blood. Magnetic resonance imaging showed a left ventricular ejection fraction of $55 \%$ (Fig. 2B-a, -b) with minimal myocardial edema over the entire left ventricular wall (Fig. 2B-c) and delayed gadolinium enhancement at the mid-inferior and septal wall (Fig. 2B-d). A transthoracic echocardiogram showed normal left ventricular dimensions and a normal systolic function through- 

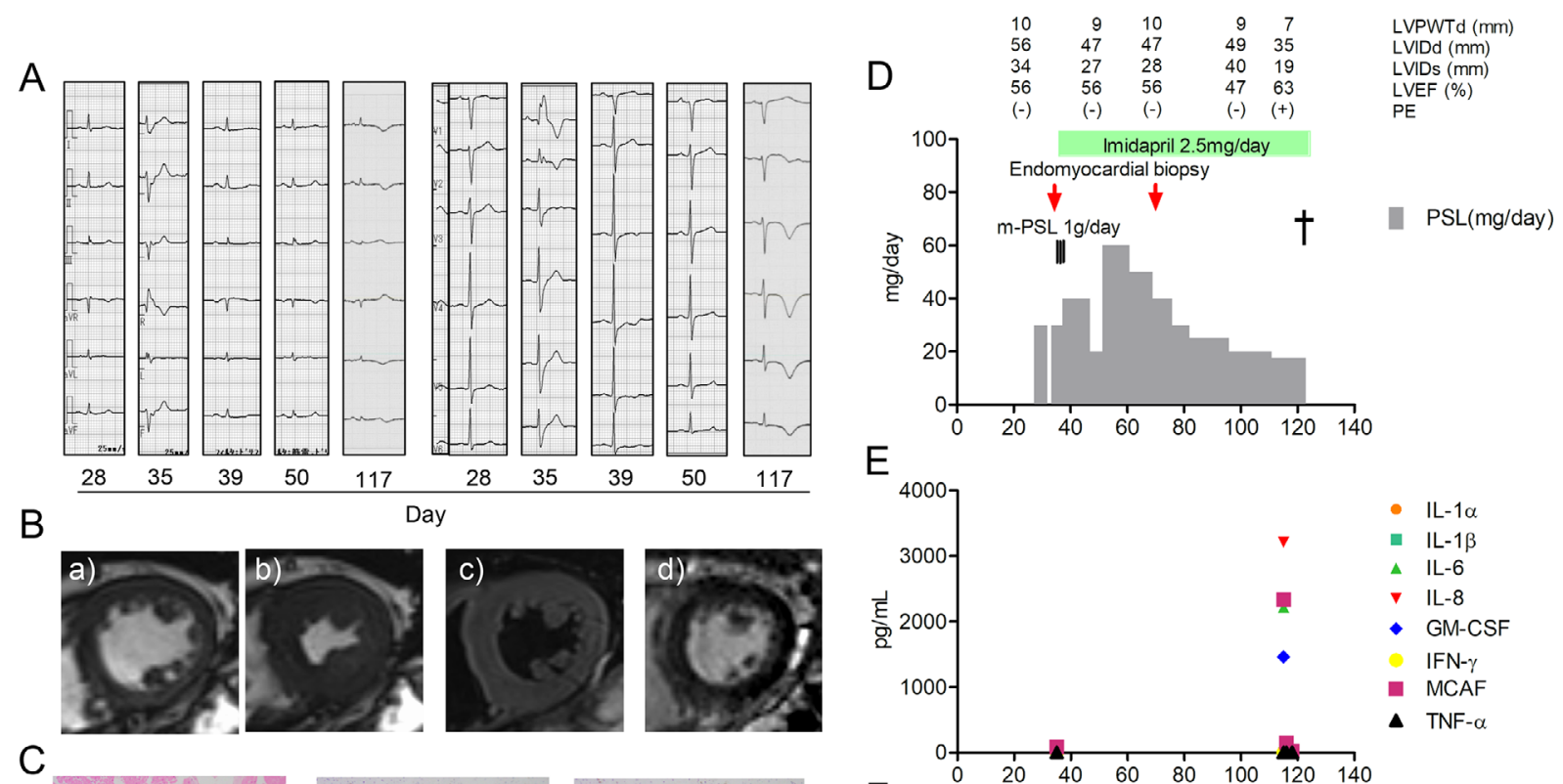
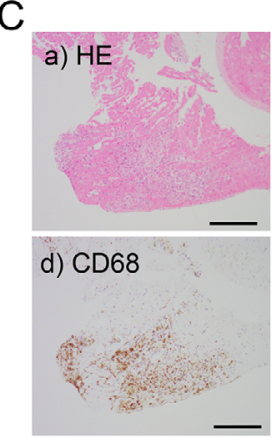
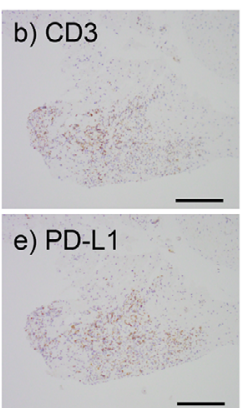

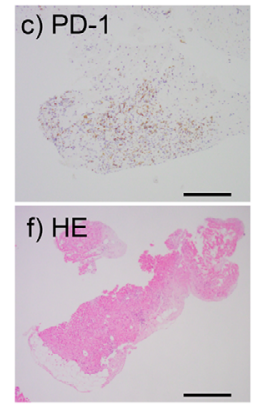

F

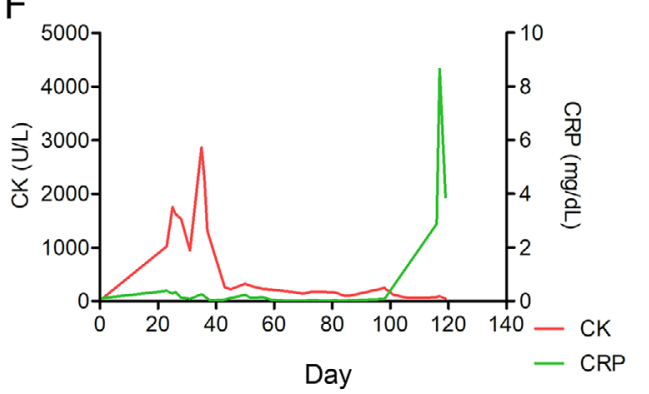

Figure 1. A: A 12-lead electrocardiograms during the clinical course in Case 1. B: Cardiac magnetic resonance imaging at diastole (a) and systole (b), dark-blood sequence for non-enhanced T2weighted imaging (c) and delayed gadolinium-enhanced imaging (d) performed at day 30. C: An immunohistochemical study using endomyocardial biopsy specimens obtained from the septal wall of the right ventricle at day 36 [a, Hematoxylin and Eosin (H\&E) staining; b, CD3 (Clone F7.2.38, DakoCytomation); c, PD-1 [anti-PD1 antibody (EPR4877 (2), ab137132, abcam)]; d, CD68 (Clone PGM1, DakoCytomation); e, PD-L1 (IHC 22C3 pharmDx, DakoCytomation)], and H\&E staining (f) in a specimen obtained at day 71. Scale bar, $200 \mu \mathrm{m}$. D: Clinical course and changes in the parameters of the left ventricular structure and function, as assessed by a transthoracic echocardiogram. E: Cytokine expressions. F: Creatine kinase (CK), and C-reactive protein (CRP) levels in the clinical course. LVPWTd: left ventricular posterior wall thickness at end-diastole, LVIDd: left ventricular diastolic internal dimension, LVIDs: left ventricular systolic internal dimension, LVEF: left ventricular ejection fraction, PE: pericardial effusion, PSL: prednisolone

out the clinical course (Fig. 2C).

He was given methylprednisolone, by adding $\gamma$-globulin for progressive thrombocytopenia, and cyclosporine for hemophagocytic syndrome (Fig. 2D). The levels of cytokines IL-6 (max, 24 pg/mL), IL-8 (max, 633 pg/mL), MCAF (max, $113 \mathrm{pg} / \mathrm{mL})$, and TNF- $\alpha$ (max, $114 \mathrm{pg} / \mathrm{mL})$ increased over time during the clinical course (Fig. 2E), but IL-1 $\alpha$, IL-1 $\beta$, and IFN- $\gamma$ were not detected. Creatine kinase levels continuously increased, and CRP showed trimodal activation despite treatment (Fig. 2F). The findings of a repeated 12lead ECG did not show changes in the ST-T segment, nor did they reveal intraventricular block or new arrhythmias. The patient died on the 57th day in the hospital because of cancer progression. Neither an endomyocardial biopsy nor autopsy was performed.

\section{Case 3}

A 63-year-old man suffering from hypopharyngeal cancer was admitted to our hospital with a high fever, leukopenia, and a decreased in appetite on the 32nd day after his fifth nivolumab (3 $\mathrm{mg} / \mathrm{kg})$ infusion. He had received one dose of docetaxel/cisplatin/5-fluorouracil, two of paclitaxel/cetuximab, and the second paclitaxel/cetuximab 12 days prior to admission. His body temperature was $38.2^{\circ} \mathrm{C}$, blood pressure $84 / 42 \mathrm{mmHg}$, heart rate 132 beats per minute, and respiratory rate 22 breaths per minute (grade 4 irAEs).

At the time of admission, blood tests showed white-blood

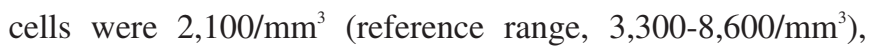



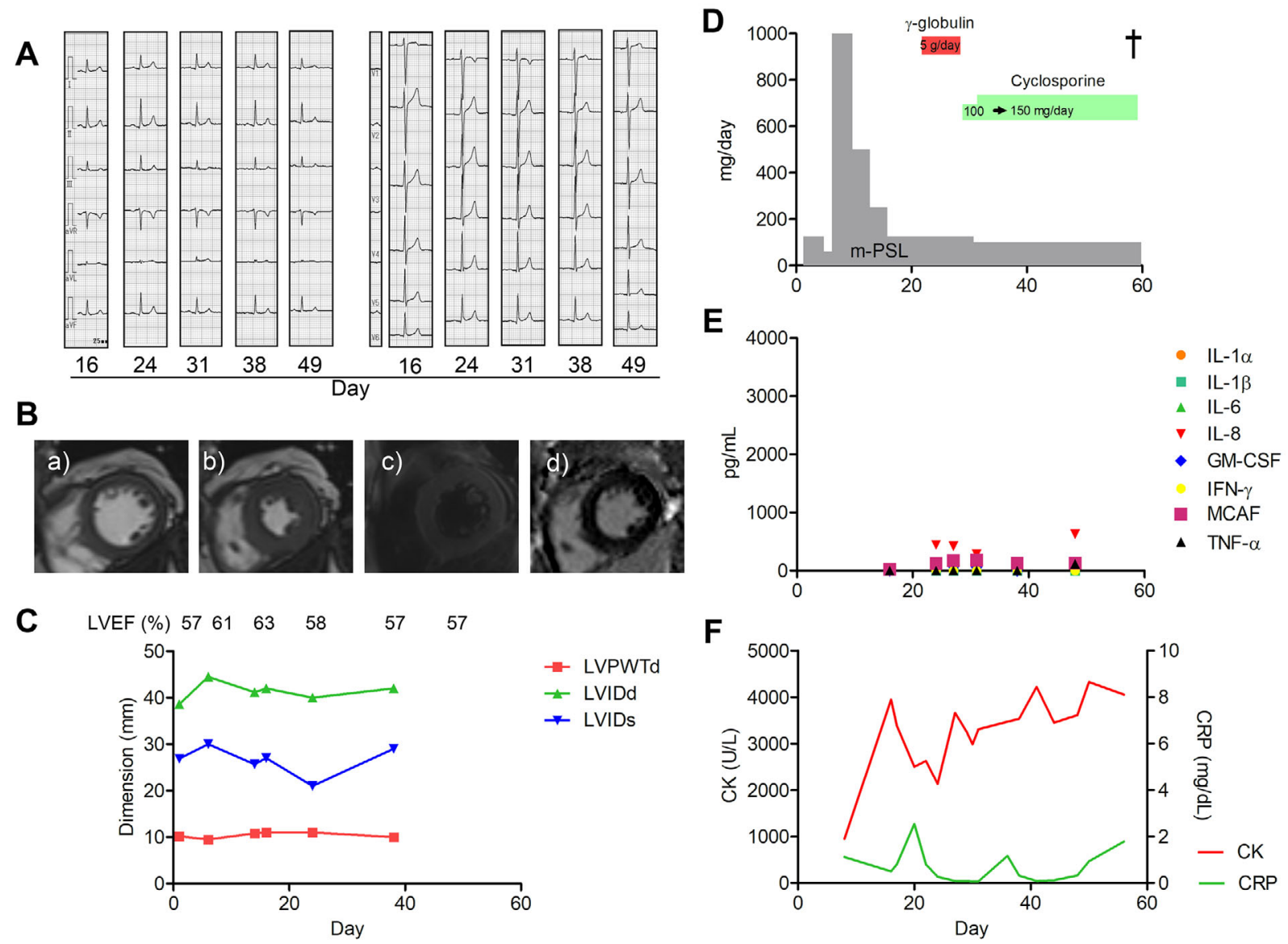

\begin{abstract}
Figure 2. A: A 12-lead electrocardiograms in Case 2. B: Cardiac magnetic resonance imaging at diastole (a) and systole (b), dark-blood sequence for non-enhanced T2-weighted imaging (c) and delayed gadolinium-enhanced imaging (d) performed at day 28. C: Changes in the parameters of the left ventricular structure and function as assessed by a transthoracic echocardiogram. D: Clinical course. E: Cytokine expressions. F: CK, and CRP levels. LVPWTd: left ventricular posterior wall thickness at end-diastole, LVIDd: left ventricular diastolic internal dimension, LVIDs: left ventricular systolic internal dimension, LVEF: left ventricular ejection fraction
\end{abstract}

neutrophils at $63.9 \%$ (reference range, $37-72 \%$ ), hemoglobin at $13.3 \mathrm{~g} / \mathrm{dL}$ (reference range, 13.7-16.8 $\mathrm{g} / \mathrm{dL}$ ), a platelet count of $134,000 / \mu \mathrm{L}$ (reference range, $158,000-348,000 / \mu \mathrm{L}$ ), creatinine at $1.03 \mathrm{mg} / \mathrm{dL}$ (reference range, $0.65-1.07 \mathrm{mg} /$ $\mathrm{dL}$ ), aspartate transaminase at $25 \mathrm{U} / \mathrm{L}$ (reference range, 13$30 \mathrm{U} / \mathrm{L}$ ), and alanine transaminase at $15 \mathrm{U} / \mathrm{L}$ (reference range, 10-42 U/L). His nivolumab concentration in the blood was $8.76 \mu \mathrm{g} / \mathrm{mL}$. His condition deteriorated with the appearance of paroxysmal atrial fibrillation. He received cardioversion.

Chest X-ray exhibited a cardiothoracic ratio of $56 \%$ and bilateral pulmonary congestion while in the supine position. Blood test findings worsened on the second hospital day with creatinine at $2.47 \mathrm{mg} / \mathrm{dL}$, aspartate transaminase at $12,422 \mathrm{U} / \mathrm{L}$, alanine transaminase at 4,321 U/L, and CRP at $8.08 \mathrm{mg} / \mathrm{dL}$. In addition, creatine kinase $894 \mathrm{U} / \mathrm{L}$ (reference range, 30-200 U/L) and cardiac troponin-T $0.16 \mathrm{ng} / \mathrm{mL}$ (reference range, $<0.014 \mathrm{ng} / \mathrm{mL}$ ) were markedly elevated at this point. The endotoxin test and serial blood culture were negative. The 12-lead ECG revealed a low-voltage R wave at the limb leads and a completely blocked right branch bundle (Fig. 3A). A transthoracic echocardiogram showed a left ventricular ejection fraction of $60 \%$, a 22-mm-thick left ventricular wall, and subsequent mild pericardial effusion (Fig. 3B).

He received extracorporeal hemoperfusion with polymyxin B (Toray Medical, Tokyo, Japan) along with continuous hemodiafiltration (cytokine-adsorbing hemofilter, AN69 ST; Baxter, Tokyo, Japan), catecholamine support, broadspectrum antibiotics, recombinant thrombomodulin, $\gamma$ globulin, and high-dose corticosteroids (Fig. 3C). Afterward, his general condition, laboratory findings, and $\mathrm{R}$ voltage normalized, and his left ventricular wall shrank to $11 \mathrm{~mm}$ in thickness. The levels of cytokines IL-6 ( $\max , 2,226 \mathrm{pg} / \mathrm{mL})$, IL-8 (max, 3,752 pg/mL), GM-CSF (max, 253 pg/mL), IFN$\gamma(\max , 247 \mathrm{pg} / \mathrm{mL}), \mathrm{MCAF}(\max , 1,465 \mathrm{pg} / \mathrm{mL})$, and CRP ( $\max , 8.08 \mathrm{mg} / \mathrm{dL}$ ) increased on the third hospital day but decreased following the treatment (Fig. 3D, E). TNF- $\alpha$ was not detected while in the hemodynamically unstable state but increased throughout recovery $(326 \mathrm{pg} / \mathrm{mL})$. IL-1 $\beta$ was not detected.

Neither an endomyocardial biopsy nor magnetic reso- 

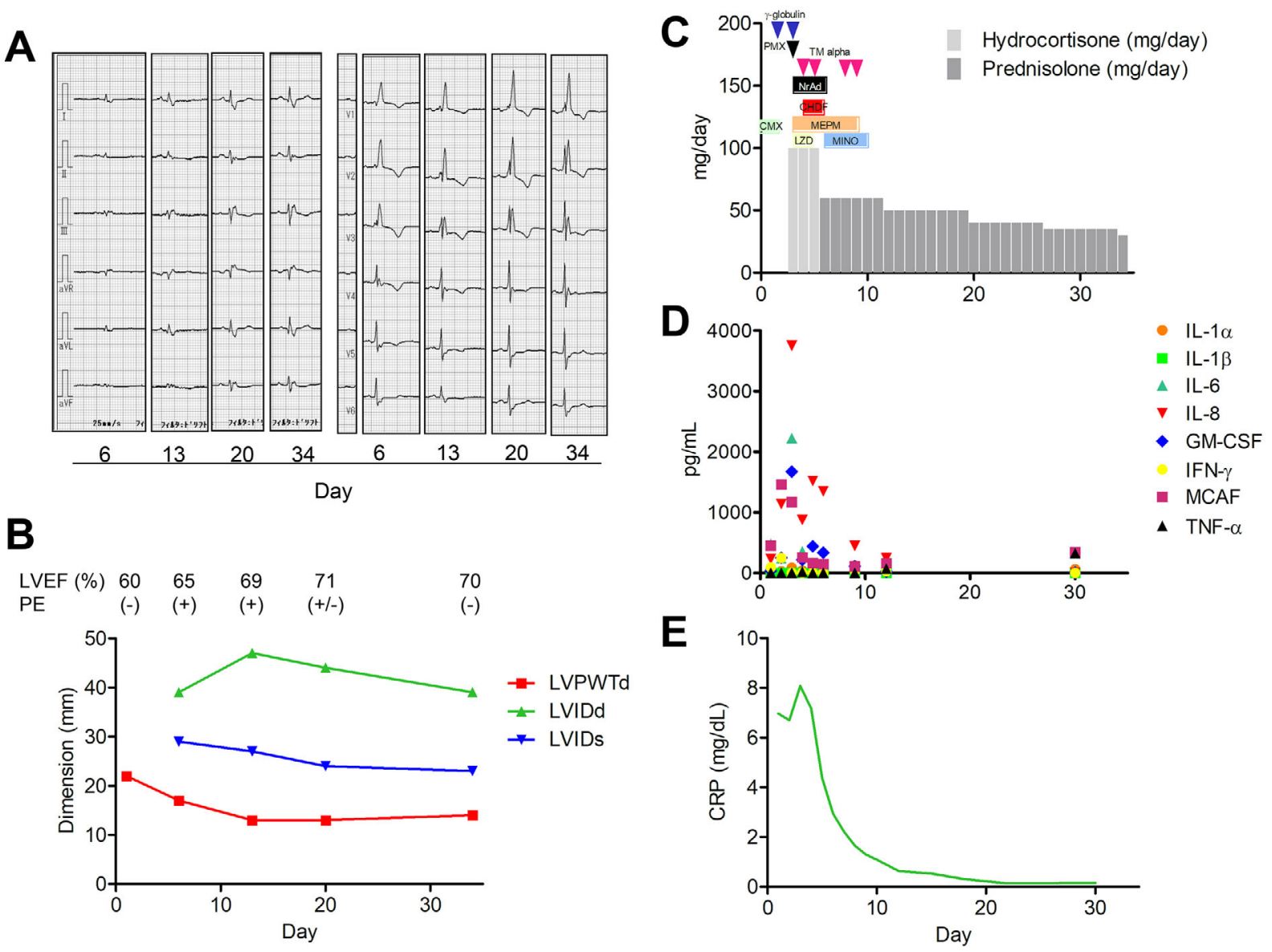

Figure 3. A: A 12-lead electrocardiograms in Case 3. B: Changes in the parameters of the left ventricular structure and function as assessed by a transthoracic echocardiogram. C: Clinical course. D: Cytokine expressions. E: CRP levels. LVPWTd: left ventricular posterior wall thickness at diastole, LVIDd: left ventricular diastolic internal dimension, LVIDs: left ventricular systolic internal dimension, LVEF: left ventricular ejection fraction, PE: pericardial effusion, PMX: polymyxin B hemoperfusion, CHDF: continuous hemodiafiltration, TM: thrombomodulin, NrAd: noradrenalin, CMX: cefmenoxime, MEPM: meropenem, LZD: linezorid, MINO: minocycline

nance imaging was performed. The patient died on the 305 th day at the affiliated hospital due to the cancer progression. No autopsy was performed.

\section{Discussion}

This report demonstrated for the first time to evaluate that cytokines were differentially expressed with cardiac complication in the individual clinical presentation after ICI therapy. The cytokine levels did not follow the course of ICIrelated myocarditis, and might be influenced by a variety of factors, such as Takotsubo cardiomyopathy, and cardiac complication of cytokine-releasing syndrome, as well as the underlying cancer, other cancer therapies, and immunosuppressive treatment.

The first case presented with biopsy-proven "definite" ICI-related myocarditis (15). Surprisingly, most cytokines were not active, despite the presence of active myocardial inflammation. It is possible for a smoldering phenotype presented little cytokine activation and/or previously administered anti-inflammatory treatment to have masked the activa- tion. However, IL-8, MCAF, IL-6, and GM-CSF selectively increased with the development of Takotsubo cardiomyopathy in the later course. Takotsubo cardiomyopathy is assumed to be the consequence of psychological stress (16) or could be another form of ICI-related cardiac toxicity (17), and IL-6 is activated in the disorder (14). The second case presented clinically "possible" myocarditis (15), and that IL8 was the predominant cytokine among the eight cytokines examined. It is likely that IL-8 level reflects cancer progression (18), but not to myocardial structure and function. The third case presented with high fever, was hemodynamically unstable, and required vasopressor support. IL-1 $\alpha$, IL-6, IL8, GM-CSF, and INF- $\gamma$ were all substantially elevated and exacerbated hemodynamic abnormalities and exerted transient left ventricular hypertrophy (possibly edema). We defined this case as cardiac complication of cytokine-releasing syndrome, and it can be triggered by various factors, including ICIs (19-21). Hemodiafiltration, to absorb excess cytokines, seemed an effective treatment to decline the cytokine concentrations in advance of an improved clinical presentation. In this case, CRP levels paralleled with IL-6 and IL-8 
concentrations $(19,22)$. TNF- $\alpha$ increased during the recovery phase, consistent with a report by Oda et al. (23), who also reported that TNF- $\alpha$ increased during the recovery of cytokine-releasing syndrome after nivolumab infusion. However, the role of TNF- $\alpha$ plays as an anti-inflammatory response in a host defense remains unclear (24). Taken together, mechanism of cytokine activation may differ between ICI-related myocarditis, Takotsubo cardiomyopathy, and cardiac complication of cytokine releasing syndrome. Changes in serum IL-8 levels reflect tumor response to ICI therapy (18). IL-8 was the predominant cytokine in our three patients, and our data suggest that the levels may reflect not only tumor burden but also severity of cardiac complications after ICI therapy.

Creatine kinase-MB isoenzyme and troponin-T levels were exceedingly elevated regardless of cardiac function and damage in the second patient. Troponin-T may not be heartspecific in the context of ICI therapy, especially not in cases of highly elevated creatine kinase $(15,25-27)$. One may consider if the elevated troponin- $\mathrm{T}$ as the result of concomitant myositis (15), myocyte toxic effects of proinflammatory cytokines (28), or ectopic production from cancer cells (29). Chen et al. (30) also reported that spreading cancer cells express cardiac troponin-I. We measured ICI concentration if they may affect the occurrence of irAEs. However, concentrations of pembrolizumab and nivolumab on the day of admission were demonstrated to be lower than expected, according to the pharmacokinetic model $(31,32)$. There are no reports that serum ICI concentration correlates with efficacy or adverse effects, and our data suggests it does not directly predict the occurrence of irAEs. Management of irAEs is recommended based on anecdotal evidence and the life-threatening nature of cardiovascular complications (33). We continued to administer the prednisolone without adding other drugs or anti-thymoglobulin after recognizing the sustained myocardial inflammation at the second biopsy of the first case. Our experience indicates that the repeated histological information might provide chance to reconsider the strategy to treat the ICI-related myocarditis. However, it is unclear whether cytokine levels are useful for predicting, or managing the cardiac complications after ICI therapy.

\section{Conclusion}

We described the time-sequential cytokine expressions of three cases who developed myocarditis, Takotsubo cardiomyopathy, and cardiac complications of cytokine-releasing syndrome after ICI therapy. We did not mention the cytokine levels before ICI therapy, but IL-8 appears to reflect the disease severity of tumor progression and respective cardiac complications. The conclusions that can be drawn from this case series are very limited, and further studies will be needed in order to explore the utility of cytokine activation for monitoring patients after ICI therapy.

This study was approved by the Human Investigation Review
Committee of the University of Miyazaki and conforms with the principles outlined in the Declaration of Helsinki, as revised in 2013. We state that, because the patients have now passed away, written informed consent was obtained from their relatives (case 1 , son; case 2, mother; case 3, sister), for the publication of this case report.

\section{The authors state that they have no Conflict of Interest (COI).}

\section{Financial Support}

This study was supported by a Grant-in-Aid for Clinical Research from Miyazaki University Hospital.

\section{References}

1. Berraondo P, Sanmamed MF, Ochoa MC, et al. Cytokines in clinical cancer immunotherapy. Br J Cancer 120: 6-15, 2019.

2. Turner MD, Nedjai B, Hurst T, Pennington DJ. Cytokines and chemokines: at the crossroads of cell signalling and inflammatory disease. Biochim Biophys Acta 1843: 2563-2582, 2014.

3. Barnes PJ. The cytokine network in asthma and chronic obstructive pulmonary disease. J Clin Invest 118: 3546-3556, 2008.

4. Friedrich M, Pohin M, Powrie F. Cytokine networks in the pathophysiology of inflammatory bowel disease. Immunity 50: 9921006, 2019.

5. Siebert S, Tsoukas A, Robertson J, McInnes I. Cytokines as therapeutic targets in rheumatoid arthritis and other inflammatory diseases. Pharmacol Rev 67: 280-309, 2015.

6. Sadreddini S, Baradaran B, Aghebati-Maleki A, Shanehbandi D, Fotouhi A, Aghebati-Maleki L. Immune checkpoint blockade opens a new way to cancer immunotherapy. J Cell Physiol 234: 8541-8549, 2019.

7. Varricchi G, Galdiero MR, Tocchetti CG. Cardiac toxicity of immune checkpoint inhibitors: cardio-oncology meets immunology. Circulation 136: 1989-1992, 2017.

8. Lim SY, Lee JH, Gide TN, et al. Circulating cytokines predict immune-related toxicity in melanoma patients receiving anti-PD-1based immunotherapy. Clin Cancer Res 25: 1557-1563, 2019.

9. Tajiri K, Ieda M. Cardiac complications in immune checkpoint inhibition therapy. Front Cardiovasc Med 6: 3, 2019.

10. Mahmood SS, Fradley MG, Cohen JV, et al. Myocarditis in patients treated with immune checkpoint inhibitors. J Am Coll Cardiol 71: 1755-1764, 2018.

11. Norwood TG, Westbrook BC, Johnson DB, et al. Smoldering myocarditis following immune checkpoint blockade. J Immunother Cancer 5: 91, 2017.

12. Johnson DB, Balko JM, Compton ML, et al. Fulminant myocarditis with combination immune checkpoint blockade. N Engl J Med 375: 1749-1755, 2016.

13. Matsumori A, Yamada T, Suzuki H, Matoba Y, Sasayama S. Increased circulating cytokines in patients with myocarditis and cardiomyopathy. Br Heart J. 72: 561-566, 1994.

14. Scally C, Abbas H, Ahearn T, et al. Myocardial and systemic inflammation in acute stress-induced (Takotsubo) cardiomyopathy. Circulation 139: 1581-1592, 2019.

15. Bonaca MP, Olenchock BA, Salem JE, et al. Myocarditis in the setting of cancer therapeutics: proposed case definitions for emerging clinical syndromes in cardio-oncology. Circulation 140: 80-91, 2019.

16. Cammann VL, Sarcon A, Ding KJ, et al. Clinical features and outcomes of patients with malignancy and takotsubo syndrome: observations from the international takotsubo registry. J Am Heart Assoc. 8: e010881, 2019. 
17. Lyon AR, Yousaf N, Battisti NML, Moslehi J, Larkin J. Immune checkpoint inhibitors and cardiovascular toxicity. Lancet Oncol 19: e447-e458, 2018.

18. Sanmamed MF, Perez-Gracia JL, Schalper KA, et al. Changes in serum interleukin-8 (IL-8) levels reflect and predict response to anti-PD-1 treatment in melanoma and non-small-cell lung cancer patients. Ann Oncol 28: 1988-1895, 2017.

19. Shimabukuro-Vornhagen A, Gödel P, Subklewe M, et al. Cytokine release syndrome. J Immunother Cancer 6: 56, 2018.

20. Rotz SJ, Leino D, Szabo S, Mangino JL, Turpin BK, Pressey JG. Severe cytokine release syndrome in a patient receiving PD-1directed therapy. Pediatr Blood Cancer 64: 2017.

21. Honjo O, Kubo T, Sugaya F, et al. Severe cytokine release syndrome resulting in purpura fulminans despite successful response to nivolumab therapy in a patient with pleomorphic carcinoma of the lung: a case report. J Immunother Cancer 7: 97, 2019.

22. Fishman JA, Hogan JI, Maus MV. Inflammatory and infectious syndromes associated with cancer immunotherapies. Clin Infect Dis 69: 909-920, 2019.

23. Oda $\mathrm{H}$, Ishihara $M$, Miyahara $Y$, et al. First case of cytokine release syndrome after nivolumab for gastric cancer. Case Rep Oncol 12: 147-156, 2019.

24. Rigato O, Ujvari S, Castelo A, Salomão R. Tumor necrosis factor alpha (TNF-alpha) and sepsis: evidence for a role in host defense. Infection 24: 314-318, 1996.

25. Messner B, Baum H, Fischer P, Quasthoff S, Neumeier D. Expression of messenger RNA of the cardiac isoforms of troponin $\mathrm{T}$ and I in myopathic skeletal muscle. Am J Clin Pathol 114: 544-549, 2000.

26. Delahunt B, Lewis ME, Pringle KC, Wiltshire EJ, Crooke MJ. Serum creatine kinase levels parallel the clinical course for rhabdo- myomatous wilms tumor. Am J Clin Pathol 116: 354-359, 2001.

27. Annesley TM, McKenna BJ. Ectopic creatine kinase MB production in metastatic cancer. Am J Clin Pathol 79: 255-259, 1983.

28. Ostermann M, Ayis S, Tuddenham E, et al. Cardiac troponin release is associated with biomarkers of inflammation and ventricular dilatation during critical illness. Shock 47: 702-708, 2017.

29. Tsuruda T, Sato Y, Kajihara K, et al. Non-canonical expression of cardiac troponin- $\mathrm{T}$ in neuroendocrine ethmoid sinus carcinoma following immune checkpoint blockade. Front Cardiovasc Med 6: 124, 2019.

30. Chen C, Liu JB, Bian ZP, et al. Cardiac troponin I is abnormally expressed in non-small cell lung cancer tissues and human cancer cells. Int J Clin Exp Pathol 7: 1314-1324, 2014.

31. Bajaj G, Wang X, Agrawal S, Gupta M, Roy A, Feng Y. Modelbased population pharmacokinetic analysis of nivolumab in patients with solid tumors. CPT Pharmacometrics Syst Pharmacol 6: 58-66, 2017.

32. Ahamadi M, Freshwater T, Prohn M, et al. Model-based characterization of the pharmacokinetics of pembrolizumab: a Humanized Anti-PD-1 monoclonal antibody in advanced solid tumors. CPT Pharmacometrics Syst Pharmacol 6: 49-57, 2017.

33. Brahmer JR, Lacchetti C, Schneider BJ, et al. Management of immune-related adverse events in patients treated with immune checkpoint inhibitor therapy: american society of clinical oncology clinical practice guideline. J Clin Oncol 36: 1714-1768, 2018.

The Internal Medicine is an Open Access journal distributed under the Creative Commons Attribution-NonCommercial-NoDerivatives 4.0 International License. To view the details of this license, please visit (https://creativecommons.org/licenses/ by-nc-nd/4.0/).

(C) 2021 The Japanese Society of Internal Medicine

Intern Med 60: 423-429, 2021 